\title{
Amplification of pico-scale DNA mediated by bacterial carrier DNA for small-cell-number transcription factor ChIP-seq
}

\author{
Janus S Jakobsen ${ }^{1,2,3}$, Frederik O Bagger ${ }^{1,2,3,4 \dagger}$, Marie S Hasemann ${ }^{1,2,3 \dagger}$, Mikkel B Schuster ${ }^{1,2,3}$, \\ Anne-Katrine Frank $k^{1,2,3}$, Johannes Waage ${ }^{1,2,3,4,5}$, Kristoffer Vitting-Seerup ${ }^{4}$ and Bo T Porse 1,2,3* $^{*}$
}

\begin{abstract}
Background: Chromatin-Immunoprecipitation coupled with deep sequencing (ChIP-seq) is used to map transcription factor occupancy and generate epigenetic profiles genome-wide. The requirement of nano-scale ChIP DNA for generation of sequencing libraries has impeded ChIP-seq on in vivo tissues of low cell numbers.

Results: We describe a robust, simple and scalable methodology for ChIP-seq of low-abundant cell populations, verified down to 10,000 cells. By employing non-mammalian genome mapping bacterial carrier DNA during amplification, we reliably amplify down to $50 \mathrm{pg}$ of ChIP DNA from transcription factor (CEBPA) and histone mark (H3K4me3) ChIP. We further demonstrate that genomic profiles are highly resilient to changes in carrier DNA to ChIP DNA ratios.

Conclusions: This represents a significant advance compared to existing technologies, which involve either complex steps of pre-selection for nucleosome-containing chromatin or pre-amplification of precipitated DNA, making them prone to introduce experimental biases.
\end{abstract}

Keywords: ChIP-seq, Pico-scale, Histone mark, Transcription factor, Epigenetic profile

\section{Background}

Genomic mapping of histone modifications, their writers, readers and erasers as well as transcription factors (TFs) has become a house-hold approach to study the genomewide regulation of gene expression programs $[1,2]$. The most widely applied method to generate such global mapping data is Chromatin ImmunoPrecipitation coupled with high-throughput sequencing (ChIP-seq), which however generally requires millions of cells as input material (e.g. [3]). Scarcity of cells in distinct, isolated in vivo populations such as phenotypically defined hematopoietic stem and progenitor cells has thus hampered direct experimentation on these. Examining such sub-populations is of intense interest for the elucidation of mechanisms governing lineage choice and commitment as well as transcriptional

\footnotetext{
* Correspondence: bo.porse@finsenlab.dk

${ }^{\dagger}$ Equal contributors

'The Finsen Laboratory, Rigshospitalet, Faculty of Health Sciences, University of Copenhagen, Ole Maaløes Vej 5, 2200 Copenhagen, Denmark

${ }^{2}$ Biotech Research and Innovation Centre (BRIC), University of Copenhagen,

Ole Maaløes Vej 5, 2200 Copenhagen, Denmark

Full list of author information is available at the end of the article
}

de-regulation in disease. Some researchers have cultured harvested cells to achieve sufficient cell numbers and performed ChIP-seq on cells undergoing differentiation in vitro, but this approach can give rise to biases due to culture conditions (e.g. [4]). Recent advances in the methodology have demonstrated successful ChIP-seq on very limited cell numbers $[5,6]$. However, these techniques are impeded by additional rounds of pre-amplification, potentially making them prone to introduce artifacts. One of these methods was established on cultured cells [6], while the other is limited to showing ChIP-seq with antibodies against histone modifications [5], yielding significantly more immunoprecipitated DNA than ChIP with antibodies to transcription factors. Furthermore, the complexity and cost of pre-amplification deters the implementation of the existing methods in many laboratories. Another recent method uses an elegant step of pre-ChIP indexing of Histone 3 containing chromatin fragments, allowing downstream distinction of input material [7]. The mixed inputs may thus function as mutual carriers during single-tube, small cell number ChIP. Importantly, the indexing step selects against nucleosome-poor genomic 
regions, making this approach less useful for unbiased investigation of genome-wide TF occupancy.

Here, we describe a straightforward and versatile workflow for both transcription factor and histone mark ChIPseq on low abundance cell populations isolated directly from the in vivo setting. A key element is the introduction of bacterial carrier DNA at the amplification step. This eliminates the previous need for pre-amplification and makes possible robust generation of sequencing libraries from picogram amounts of ChIP DNA.

\section{Results}

Histone mark ChIP-seq of hematopoietic cell populations

The scarcity of biologically relevant in vivo material is often barring global level investigations into normal development as well as the aberrant regulation behind cancer and other complex diseases. Of particular interest are the genomewide binding patterns of transcription factors and the associated epigenetic profiles, which may pinpoint aberrant molecular mechanisms underlying transcriptional dysregulation and development of disease. Here, we use a standard FACS regimen (Additional file 1: Figure S1) to isolate a specific hematopoietic GMP-blast population from Cebpa ${ }^{p 30 / p 30}$ mice expressing a truncated variant of the myeloid transcription factor CEBPA [8]. These mice develop acute myeloid leukemia with complete penetrance, and have been studied in detail [9-12]. However, the precise molecular dysregulation driving leukemogenesis remains obscure. We therefore developed a ChIP-seq assay compatible with the numbers of isolated leukemic cells from the in vivo context.
First, we optimized our ChIP protocol for small cell numbers, which is described in detail here for clarity. Immediately after the sorting procedure, isolated cells were exposed to formaldehyde for cross-linking chromatinassociated proteins to the DNA, washed and snap frozen in liquid nitrogen. Next, they were subjected to sonication to break the chromatin into suitably sized fragments (Figure 1 and Methods). We found that careful inspection of the DNA size distribution of each batch of chromatin was useful to prevent further processing of low quality samples. This was achieved either by processing a parallel sample of c-Kit enriched BM cells, providing a sufficient cell number for standard gel electrophoresis, or by direct inspection of each sample using the Bioanalyzer DNA1000 assay (Methods and (Additional file 2: Figure S2)). Chromatin from roughly 125,000 cells, equivalent to $250-300 \mathrm{ng}$ of naked DNA, was used as input for each ChIP experiment with antibodies against the histone marks H3 Lys27 trimethylation (H3K27me3) or H3 Lys4 trimethylation (H3K4me3), performed in siliconized tubes with optimized washing conditions and titrated antibody and antibody-binding beads (Methods). Utilizing a thorough approach of extended protein degradation and de-crosslinking steps, as well as phenol-chloroform extraction for retrieving ChIP DNA ensured robust high recovery. This approach allowed us to effectively enrich for genomic sequences associated with either H3K27me3 or H3K4me3 as assessed by quantitative PCR (qPCR) (Additional file 3: Figure S3). The H3K27me3 ChIP produced ca. 2 ng of DNA for each sample. By making minor but important changes to the standard Illumina protocol, we were able to consistently amplify the 2 ng ChIP DNA to generate libraries for high-throughput

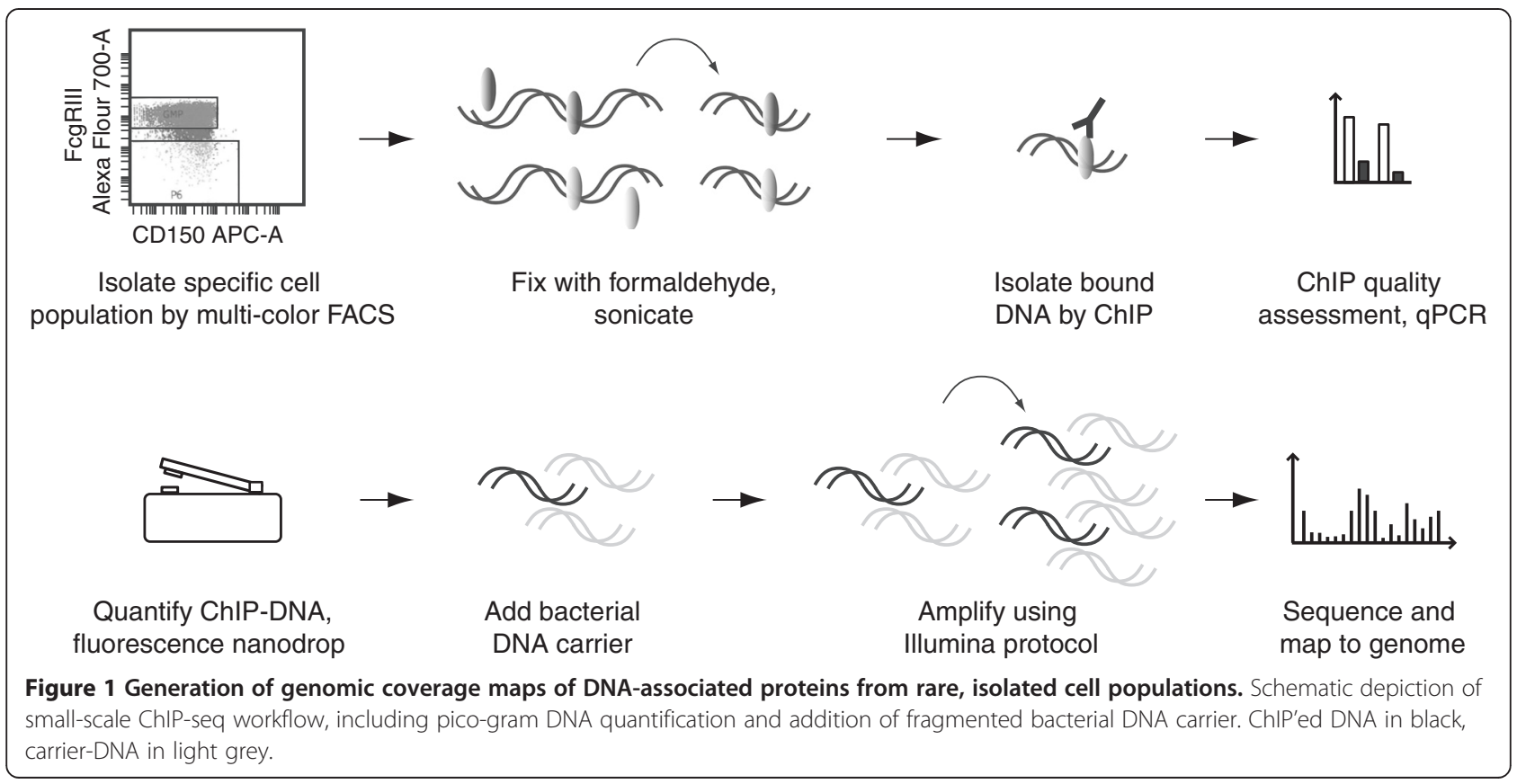


sequencing (Methods). The H3K4me3 ChIP yielded an amount of DNA below the effective range of standard absorbance or fluorescence assays. We circumvented this obstacle by taking advantage of the fluorescence Nanodrop instrument, which allows reliable detection of DNA down to $5 \mathrm{pg} / \mathrm{ul}$ in a $1 \mathrm{ul} \mathrm{sample} \mathrm{volume} \mathrm{(Additional} \mathrm{file} \mathrm{4:} \mathrm{Figure} \mathrm{S4).}$ With this approach, H3K4me3 ChIP DNA was measured to ca. 700 pg DNA, which we pooled to obtain the $2 \mathrm{ng}$ sufficient for robust amplification (Methods). Using the Illumina Hiseq platform, we deep sequenced two libraries derived from two biologically independent samples for each of the two histone marks (Additional file 5: Table S1). We processed the aligned reads into genomic coverage profiles using standard procedures (Methods). Visual analysis of the profiles suggested a good concordance with previous findings [5,13], showing enrichment of the H3K27me3 mark in gene bodies, intergenic regions as well as promoters and H3K4me3 in gene promoter regions (Figure 2A). A quantitative analysis mapped $\mathrm{H} 3 \mathrm{~K} 27 \mathrm{me} 3$ reads as $6 \%$ in promoter (5' proximal) and $56 \%$ in gene body locations (intronic/exonic), while $21 \%$ of $\mathrm{H} 3 \mathrm{~K} 4 \mathrm{me} 3$ reads resided in promoters (Figure 2B). Promoter H3K4me3 modifications were positively and H3K27me3 negatively correlated with activity of associated genes, as observed previously (e.g. [5,13-17]) (Figure 2C). Finally, we assessed the reproducibility of our ChIP-seq approach by comparing coverage in promoter regions from two biologically independent replicates and found a high degree of correspondence, both by visual inspection (example in Figure $2 \mathrm{~A}$ and $\mathrm{E}$ ) and quantitative measures (Figure 2D). In the examined cell type, we observe a near mutually exclusive pattern of H3K4me3 and H3K27me3 marks in promoters (Figure 2E), as opposed to e.g. embryonic stem cells displaying a set of doublemarked promoters at poised genes, a hall-mark of the undifferentiated state $[18,19]$.

\section{Transcription factor ChIP-seq of specific, isolated cell populations}

Chromatin-associated proteins with limited genomic distributions generally yield less ChIP DNA when compared

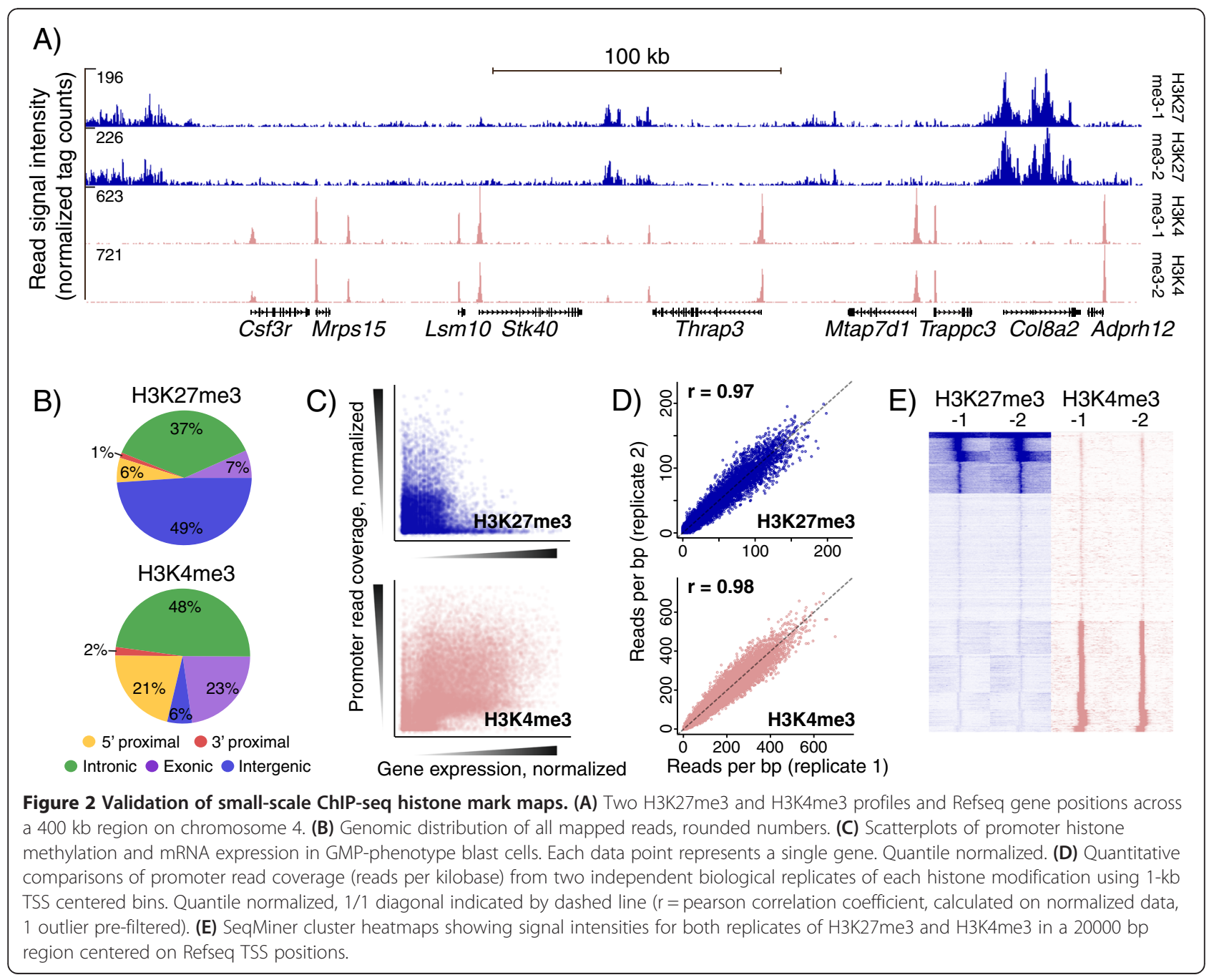


to chromatin components showing broader distributions. This is in line with our observations for H3K4me3 versus H3K27me3 modifications, with characteristic peak-like and broad distributions, respectively (e.g. [13]). Accordingly, we expected transcription factor ChIP to yield even less output DNA. To address this issue we used a larger input cell number. We performed ChIP with antibodies against CEBPA, a transcription factor known to be expressed in the GMP blast population [10], using chromatin from 250,000 or 500,000 cells, corresponding to 500-1000 ng input DNA per sample. By performing qPCR for amplicons covering known CEBPA target sequences, we verified the quality of the immuno-precipitation step (Additional file 3: Figure S3). Utilizing the fluorescent nanodrop, we measured the ChIP DNA yields of down to $250 \mathrm{pg}$ (250,000 cells), an amount prohibitive for standard use as a starting point for Illumina amplification.

We reasoned that an absolute loss during the amplification procedure could be ameliorated by adding a DNA carrier. First, we set out to test the use of a synthetic 120-mer oligo devoid of the terminal 3'-OH group, which would prevent ligation to the amplification adaptors and hence preclude amplification during the PCR step. This gave rise to unacceptable biases as displayed by non-linear amplification of CEBPA enriched regions (data not shown), probably due to low complexity of the DNA pool during PCR amplification. We next hypothesized that addition of complex carrier DNA that could be amplified would be ideally suited to surpass this problem. Hence, we performed an in silico mappability test of 10 million 50-bp randomly extracted $E$. coli sequences, of which 0 mapped to the mouse genome and 14457 $(<0.15 \%)$ to the human counterpart (Additional file 6: Additional Methods). Adding fragmented bacterial DNA $(1700 \mathrm{pg}$ ) to the CEBPA ChIP DNA (ca. $300 \mathrm{pg}$ ) produced the $2 \mathrm{ng}$ established to perform robustly in amplification. By deep sequencing the resulting compound library, we were able to map up to $20 \%$ of the obtained sequences to the mouse genome (Additional file 5: Table S1). This corresponded with the ratio between ChIP DNA and bacterial carrier and typical ChIP-seq mapping frequencies. CEBPA peaks were observed in promoter proximal positions characteristic of many transcription factors (e.g. [20]) (Figure 3A). Quantitative assessment of CEBPA peak positions revealed a genomic distribution analogous to a thoroughly validated CEBPA ChIP-seq dataset obtained with liver cells (Figure 3B) [21]. Further visual inspection identified several examples of CEBPA peaks shared between hepatocyte and myeloid progenitor data sets (Figure 3C). Strikingly, the two archetypical homeostatic liver genes (Albumin (Alb) and CarbamoylPhosphate Synthase 1 (Cps1)) displayed CEBPA peaks in hepatocytes, but not in myeloid cells. Conversely, many genes characteristic of the myeloid lineage displayed
CEBPA peaks only in the myeloid ChIP-seq dataset (e.g. Myeloperoxidase (Mpo), Colony stimulating factor 3 receptor (Granulocyte)(Csf3r), Matrix Metallopeptidase 8 (Neutrophil Collagenase)(Mmp8), Selectin L(Sell), Colony Stimulating Factor 2 (Granulocyte-Macrophage) (Csf2), Fc Fragment of IgG, Low Affinity IIIb, Receptor (Fcgr3)) (Figure 3C). Several of these are known CEBPA targets. Two additional pieces of evidence supported the validity and specificity of our CEBPA genomic occupancy data. Firstly, the top hit of a de novo motif search in the enriched sequences matched the known CEBPA binding logo (Figure 3D), and this motif was found strongly enriched in the peak centers (Figure 3E). Secondly, robust conservation in these sequences were centered on CEBPA motifs, implying functional evolutionary constrain (Figure 3F). Visual and quantitative comparisons of coverage from two biologically independent repeats indicated a high degree of reproducibility (Figure 3A, G, H). Collectively, these data provide evidence that transcription factor ChIP-seq on smallcell-number populations is possible using bacterial DNA as a carrier during the amplification step.

\section{Amplification from picogram amounts of ChIP DNA}

Many immunophenotypically defined hematopoietic compartments, e.g. the hematopoietic stem cells, consist of a very limited number of cells. ChIP from small cell populations, as has been done previously by several groups [3,5,6,22-24], consistently yield very limited quantities of ChIP DNA. Hence, we wanted to investigate if our carrier DNA amplification approach could be applied on picogram-scale amounts of DNA. To test this, we generated a series of compound DNA Illumina amplified libraries with varying ratios of bacterial carrier DNA and ChIP DNA from three pooled CEBPA ChIP samples (performed on 250,000 cells each) to allow direct comparisons between libraries. Aiming to minimize the dilution ratio, some of these libraries were generated using total input amounts of 1000 or $500 \mathrm{pg}$. The resulting four libraries (CEBPA-3, 100 pg ChIP DNA and 1900 pg carrier; CEBPA-4, 100 and 900 pg; CEBPA-5, 100 and 400 pg; CEBPA-6, 50 and $450 \mathrm{pg}$ ), all displayed amplification output yields and size distributions comparable to libraries generated from $2 \mathrm{ng}$ DNA (Additional file 7: Figure S5). High-throughput sequencing of these libraries resulted in mapping frequencies close to the expected based on standard ChIPseq mapping efficiency and ratios of ChIP DNA to bacterial carrier DNA. E.g. from a read number of roughly 85 million for CEBPA-5, 8.4 million mapped uniquely to the mouse genome (Additional file 5: Table S1). Visually, genomic coverage profiles for each of the four new libraries closely matched our previous CEBPA tracks (Figure 4A). An analysis of the degree of correlation between dilute libraries and CEBPA-1 indicated consistent, 


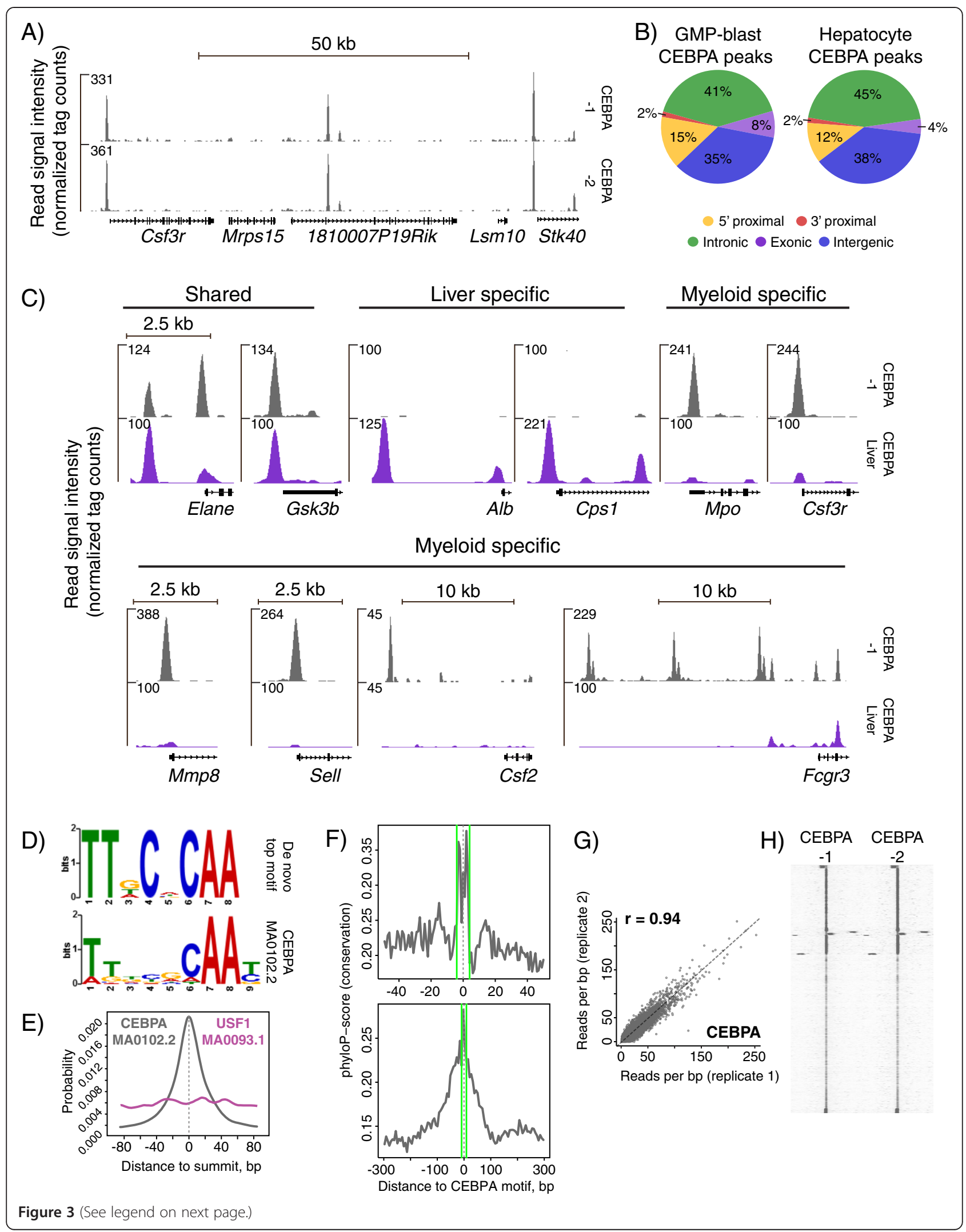


(See figure on previous page.)

Figure 3 Validation of small-scale transcription factor ChIP-seq. (A) Profiles generated from 300 pg CEBPA ChIP-DNA. Region spanning a 100 kb region on chromosome 4. (B) Genomic distribution of top 26,713 CEBPA peaks (fold 30 cut-off) from GMP-blasts compared with 7,660 CEBPA peak positions in hepatocytes (fold 30 cut-off, data from [21]), rounded numbers. (C) CEBPA coverage tracks from GMP-blast and hepatocyte gene loci of shared, specific liver or myeloid cell expression. See text for full gene names. (D) De-novo motif search output from MEME (upper panel), and top match JASPAR motif CEBPA (MA0102.2), both in logo format (lower panel). Data set as in D. (E) Distribution of the top match motif CEBPA (MA0102.2, grey) in 200 bp regions centered on CEBPA-1 peak summits. USF1 (MA0093.1, purple) included for comparison. (F) Phylo-P conservation plots in 100 bp (upper panel) and 600 bp (lower panel) regions centered on CEBPA-motifs in CEBPA-1 peaks. Green lines delineate motif position. (G) Quantitative comparison of mean read coverage of two ChIP-seq repeats, CEBPA-1 and CEBPA-2, using CEBPA-1 peak regions defined by MACS2. Quantile normalized, 1/1 diagonal indicated by dashed line ( $r=$ pearson correlation coefficient, calculated on normalized data, 1 outlier pre-filtered). MACS2 peak boundaries for CEBPA-1 (H) SeqMiner cluster heatmaps showing signal intensities for CEBPA-1 and CEBPA-2 profiles in 10000 bp regions centered on peak summits. For $F$ and $G$, peak set as in $B$.

high reproducibility (Figure $4 \mathrm{~B})$. Strikingly, the library containing least ChIP DNA (CEBPA-6, 50 pg) display a pearson correlation of 0.85 with the 300 pg CEBPA- 1 library and overall recapitulate the genomic coverage of this library (Figure 4B, C).

Next, we investigated the diluted libraries for presence of CEBPA peaks at known CEBPA targets (e.g. Nuf2, NDC80 kinetochore complex component, Sing-7 homolog, nonsense mediated $m R N A$ decay factor, CCAAT/enhancer binding protein (C/EBP), alpha and - beta, prostaglandinendoperoxide synthase 2 and interleukin 6 receptor, alpha), and found these in all coverage profiles (Figure 5A) [21].
To test our panel of libraries further, we performed qPCR to quantify amplicons corresponding to the CEBPA enriched regions mentioned above, which indicated reproducible and practically uniform amplification across dilution ratios and input amounts (Figure 5B).

\section{ChIP-seq using 10,000 cells}

To investigate if our methodology allows ChIP-seq on limited cell numbers, we transplanted a new cohort of mice with the leukemic strain also used in the above and FACSisolated batches of 10,000 GMP-blasts. We optimized the chromatin preparation procedure to ensure maximal input

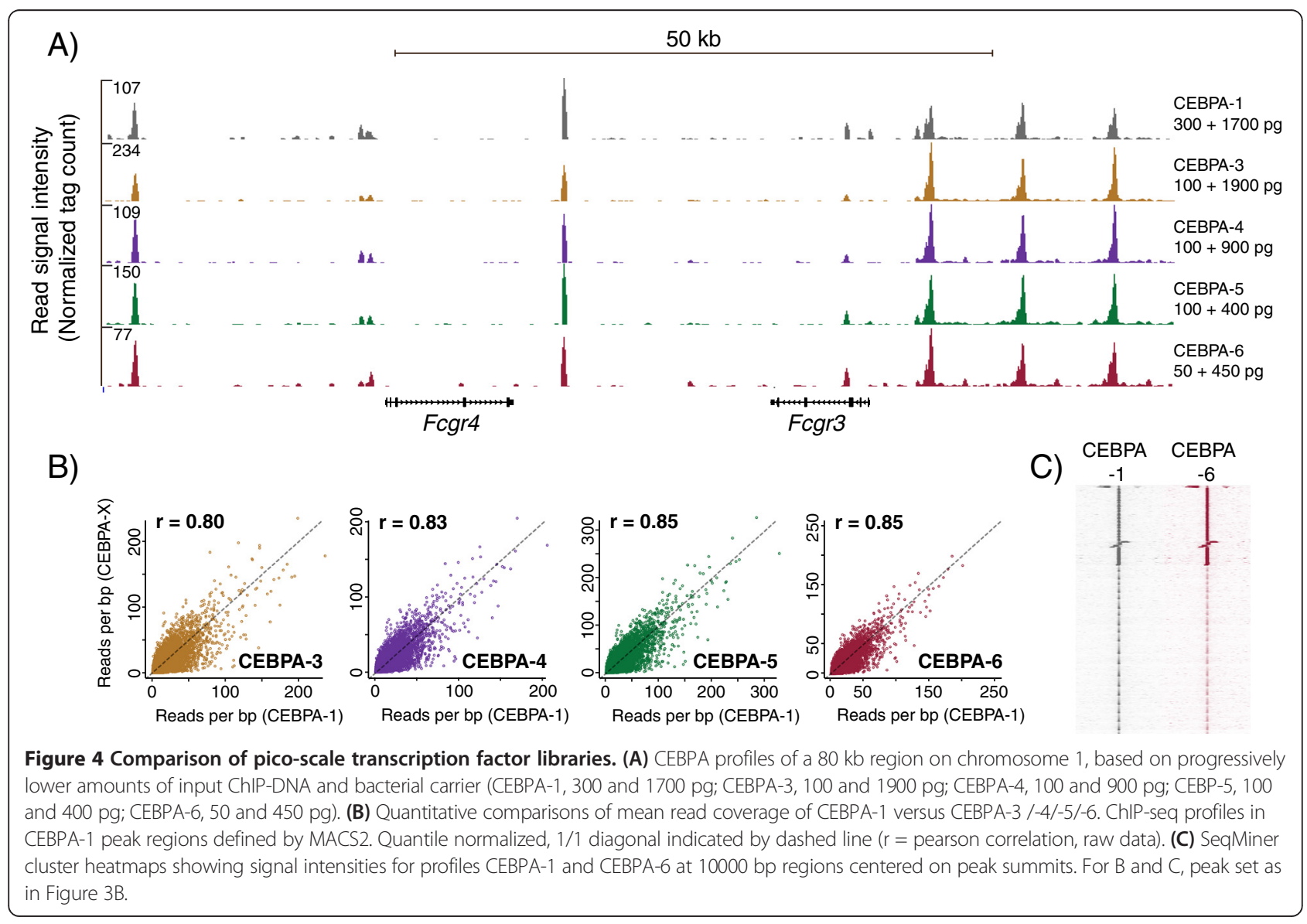




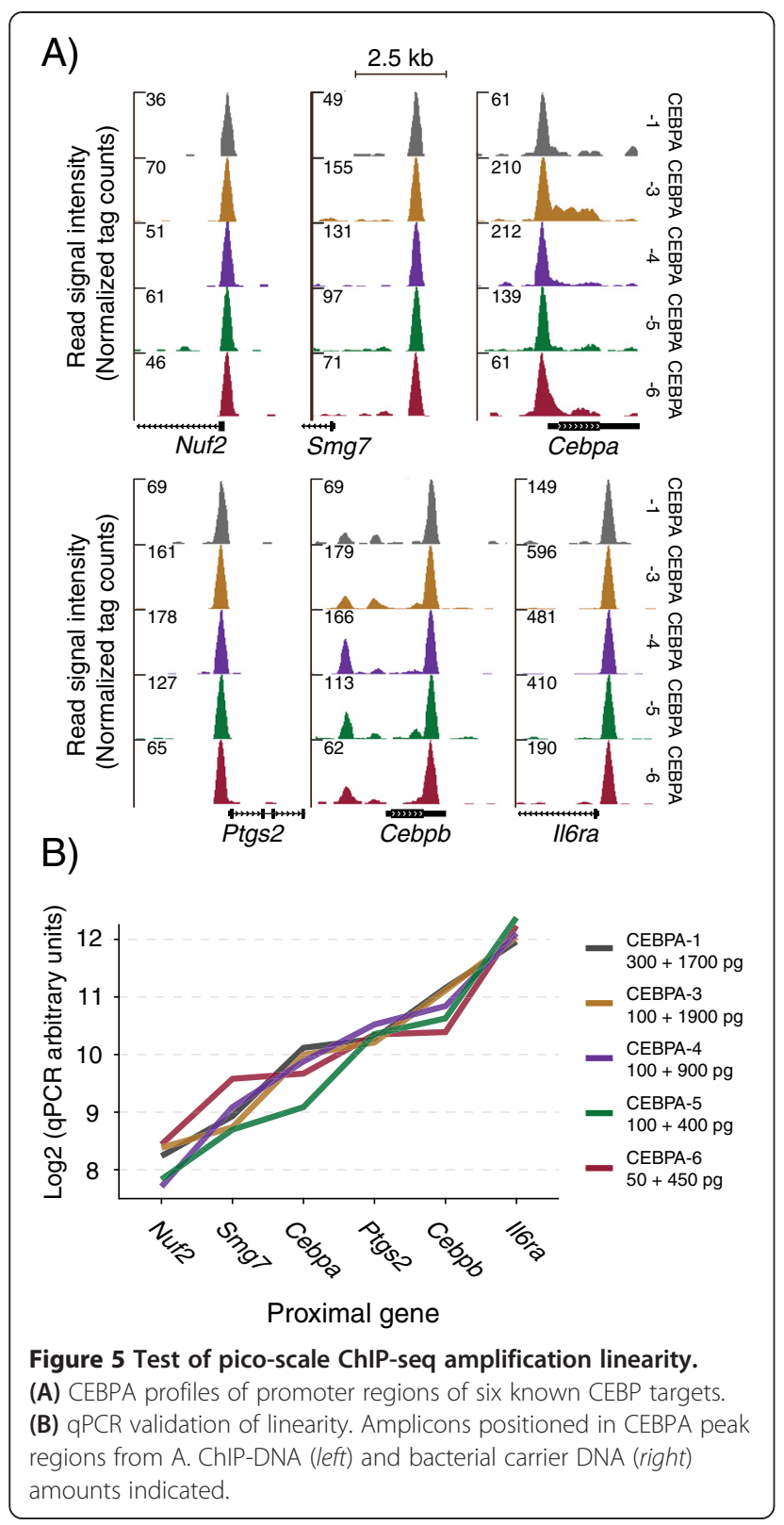

material for the small-cell-number ChIP (Additional file 8: Additional Protocols and Additional file 9: Figure S6). Thorough optimization of ChIP conditions produced robust enrichments for histone mark (H3K4me3) as well as TF (CEBPA) ChIP, both of which compared favorably to ChIPs done according to a previously published 10,000 cell ChIP methodology by Zwart et al. [24] (Figure 6A, B). The major advance in the previous study was addition of a combined mRNA/Histone carrier/blocker during the ChIP step. We amended our protocol to include such a carrier, resulting in even better enrichment as assessed by qPCR (Figure 6A, B). A small amount of 10,000 cell ChIP-DNA was used for examining enrichment and establishing quantity, leaving ca. $60 \mathrm{pg}$ DNA. Parallel generation of sequencing libraries with and without bacterial carrier demonstrated that at the $60 \mathrm{pg}$ ChIP-DNA range, carrier is required for robust production of high-quality Illumina libraries (Additional file 10: Figure S7). Both visual inspection and quantitative analyses of genomic coverage tracks from 10,000 cell ChIPs revealed good correspondence with libraries generated from biologically independent higher-cell-number ChIPs (Figure 6C, D, E). CEBPA-1 peaks were to a high degree shared with a biological repeat (CEBPA -2), the diluted library (CEBPA-6) and the low-cell-number ChIP (CEBPA-10K) (Additional file 6: Additional Methods). Importantly, the H3K4me3-10K profile generated from 60 pg ChIP-DNA, which display substantially fewer mapped reads (Additional file 5: Table S1), is highly similar to the H3K4me3-1 profile from 2 ng DNA resulting from ChIP without using bacterial carrier for amplification (Figure 6C, D).

The recent establishment of the online Next Generation Sequencing Quality Control (NGS-QC) Generator, a useful cross-platform quality assessment tool [25], allowed a quantitative comparison of our ChIP-seq approach with already published methods (Additional file 6: Additional Methods). Our methodology robustly produced NGS-QC scores matching or exceeding those from previous approaches (Figure 6F and Additional file 11: Table S2). Specifically, Shankaranarayanan (LinDA) amplification [6] resulted in considerably lower NGS-QC scores at the 10,000 cell range, while Adli amplification [5] and the Zwart methodology [24] produced data sets with comparable scores for histone modification or transcription factor ChIP-seq profiles, respectively (Figure 6F).

\section{Discussion and conclusions}

Here, we present a complete work-flow for ChIP-seq on the limited cell populations of distinct in vivo cell compartments. By isolating a defined cell population with a standard immunophenotypic sorting regimen and using a rigorous and reliable ChIP approach, we were able to enrich regions marked by both broadly (H3K27me3) and more narrowly (H3K4me3) distributed histone modifications as well as the precise regions defined by occupancy of the central hematopoietic transcription factor, CEBPA. Importantly, the fluorescence nanodrop instrument allowed us to reliably measure the obtained picogram amounts of ChIP DNA. We demonstrate that these amounts of ChIP DNA could be faithfully amplified to generate libraries for Illumina sequencing by addition of fragmented $E$. coli carrier DNA. Further, we show the resulting coverage profiles to match previous data for the actively transcribed gene associated (H3K4me3) and repressed gene associated (H3K27me3) histone marks, and to specifically recover known myeloid specific CEBPA target regions. We provide evidence that CEBPA bound regions from a pool of ChIP'ed DNA down to $50 \mathrm{pg}$ are amplified in a linear manner with 


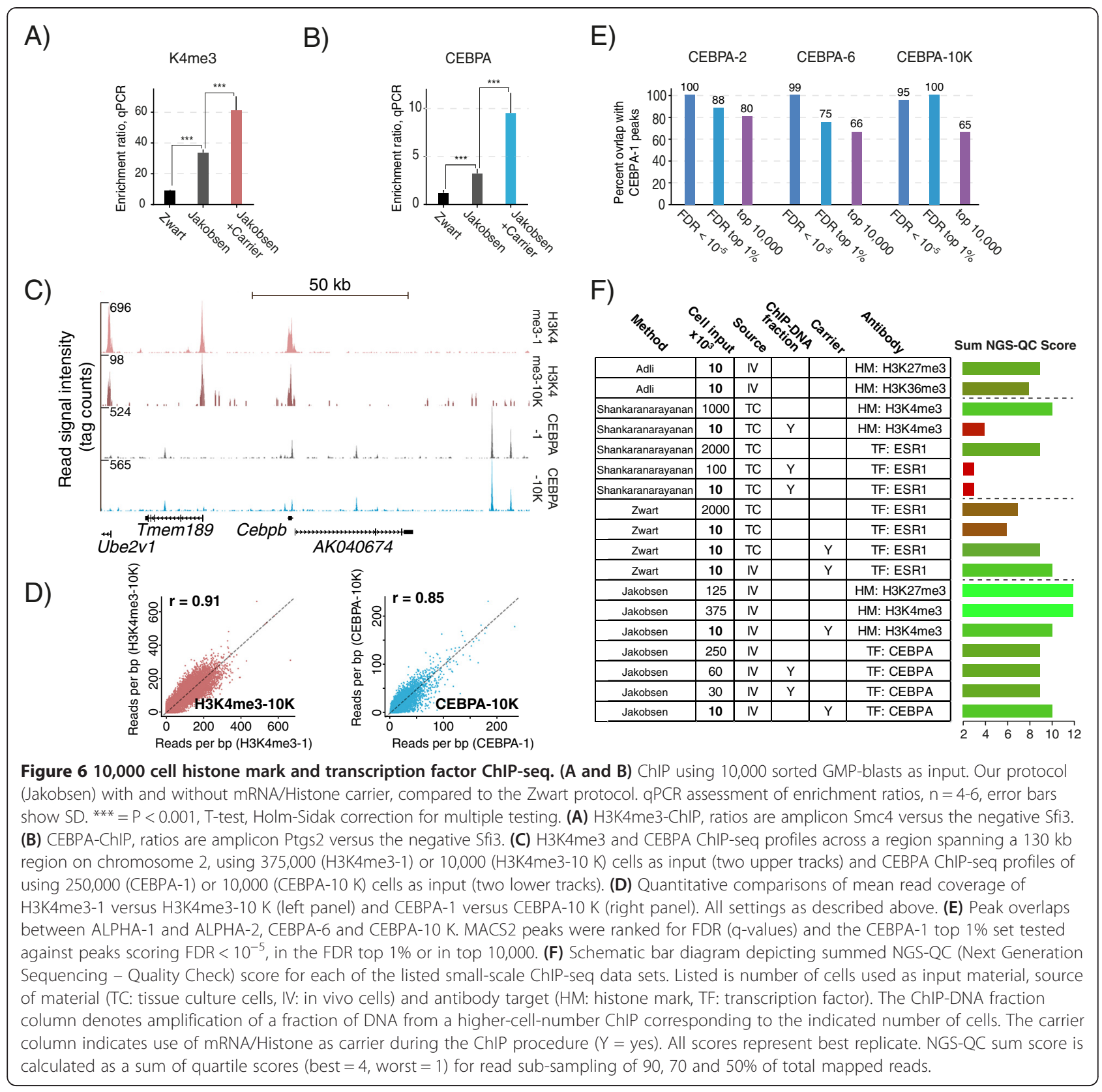

our technique. Finally, we demonstrate that our straightforward methodology can produce high-quality ChIP-seq coverage tracks from as little as 10,000 isolated in vivo cells using antibodies against histone mark (H3K4me3) as well as transcriptional factor (CEBPA) antibodies. Importantly, the H3K4me3-ChIP 10,000 cell genomic coverage profile is very similar to the profiles generated without bacterial carrier (H3K4me3-1/-2), indicating that the addition of bacterial carrier does not impede amplification or Illumina sequencing.

Our methodology can be used to elucidate important biological circuitries at a global level. For instance, we can clearly detect differences of direct targets of CEBPA in specific cell types as illustrated in Figure 3. Importantly, two recently published studies demonstrate the usefulness of our approach by revealing molecular mechanisms behind initiation of acute myeloid leukemia and regulation of hematopoietic stem cells [26,27]. Furthermore, our technology should combine easily with other genomics approaches, for instance Chia-PET [28], to permit generation of libraries from otherwise prohibitively small amounts of DNA.

Other available methodologies that allow generation of ChIP-seq data from limited amounts of input material rely on an extra amplification step, based either on custom designed random primers or T7 RNA-polymerase 
technology [5,6]. Even as these methods are very useful, introducing extra and complex steps in the amplification procedure will inevitably increase the risk of errors, and is costly and time-consuming. Our method is based on the conceptually simple addition of non-mapping DNA as carrier and should as such be easy to implement in any laboratory that already performs Illumina platform ChIP-seq.

A disadvantage of our approach is the added cost of sequencing E. coli DNA, generating data that will be discarded. This drawback increases with the ratio between bacterial and ChIP DNA, i.e. as fewer cells are used or less material recovered, for example as a result of poor antibodies or low expression of the ChIPed factor. We have tried to address this issue by demonstrating the feasibility of using just 500 picogram total input material for the amplification procedure. The steady decline of sequencing prices should also help reduce this disadvantage.

Several groups have optimized ChIP from very small cell numbers (e.g. [3]), whereas our study focusses on refining the amplification step of ChIP-seq. Some reports have shown the addition of carrier chromatin during the ChIP stage to facilitate the application on small cell numbers, but these methodologies are either not tested or incompatible with high-throughput sequencing [29]. Recently, Zwart et al. demonstrated an increase in the ChIP-seq signal to noise ratio by adding carrier consisting of RNA and histones [24]. While ChIP protocols generally include bovine serum albumin as a carrier (e.g. [30]), Zwart and coworkers speculate that combined oligonucleotide RNA and histones mimic chromatin better, and hence offer improved blocking of spurious binding. Both components are degraded prior to the amplification step, making the modification suitable for use in ChIP-seq. We compare a low-cell-number optimized version of our methodology and find it to surpass the Zwart approach for the tested antibodies (Figure 6A, B). Nevertheless, adopting the mRNA/histone ChIP level carrier considerably improves our protocol (Figure 6A, B), clearly demonstrating the value of the Zwart at al. contribution to the development of ChIP technology.

In summary, our study, in combination with the progress of sequencing technology, makes possible relatively straight-forward ChIP-seq even on very scarce cell numbers e.g. from stem cell populations. This opens the door for extensive genome-wide investigation of the regulatory circuitries at all differentiation levels of in vivo tissues.

\section{Methods}

Additional methods and protocols in additional files

(Additional file 6: Additional Methods and Additional file 8: Additional Protocols and Buffer Recipes).

\section{Mouse work}

Blast-GMP populations were generated as described previously [31]. Briefly, fetal livers were isolated from E14.5 Cebpa ${ }^{p 30 / p 30}$ (CD45.2) female embryos, homogenized and filtered through a 70 um filter. Each liver was transplanted into four lethally irradiated recipients (CD45.1) by tail vein injection. The recipients developed acute myeloid leukemia (AML) within 9-11 months after transplantation and sacrificed when moribund. The bone marrow (BM) was isolated and retransplanted into sublethally irradiated recipients $\left(1.5 \times 10^{\wedge} 6\right.$ whole BM cells/ recipient). The procedure was repeated to generate a tertiary leukemia, from which whole BM was retrieved for isolation of GMP-blasts. Genotyping was performed as previously described [10], utilizing genomic tail DNA as template. All mouse work was performed according to national and international guidelines and approved by the Danish Animal Ethical Committee under license \#201215-2934-00725.

\section{Flow cytometry and purification of hematopoietic progenitor populations}

BM cells were isolated and stained using the following antibodies: CD41-FITC, CD105-PE, Gr-1- PECy5, B220-PECy5, CD3-PECy5, Sca1-PerCp5.5, Ter119-PECy7, CD16/CD32Alexa Flour 700, c-Kit APC-Alexa 750, CD45.2-Biotin, CD45.1-eFlour450 (all from eBioscience), Mac1-PECy5 (BD Biosciences), Streptavidin-QD655 (Invitrogen), CD150-APC (BioLegend), and 7-AAD (Invitrogen) as viability marker. For sorting, cells were c-Kit enriched using CD117 microbeads and MACS LS separations columns (Miltenyi Biotec) prior to staining. GMP-blasts used in this study were defined as previously described $[8,10]$ (Additional file 1: Figure S1).

\section{Chromatin preparation}

GMP-blasts were sorted into pre-wetted siliconized microcentrifuge tubes (Biozym, cat\#1267-2970) on ice, containing $300 \mu \mathrm{l} \mathrm{PBS}$ and 3\% Fetal Calf Serum (FCS) (Saveen \& Werner, cat\#FB-1090/500). Volume was adjusted to $1.4 \mathrm{ml}$ with cold PBS-3\% FCS and samples cross-linked in 1\% formaldehyde for 10 minutes at room temperature using a rotator. Cross-linking was quenched using 0.125 M Glycine and samples washed twice in cold PBS-3\% FCS using a swinging bucket centrifuge ( 4 minutes, $600 \mathrm{~g}$ ) and soft spin settings to minimize material loss. Cells were lysed as described previously in $300 \mu$ lysis buffer [21], applying up/ down pipetting 10 times using a $100 \mathrm{ul}$ tip low retention tip. Samples were sonicated using a Bioruptor sonicator plus for 30 cycles, 15/30 seconds on/off, high setting, and debris pelleted by centrifuging cold at 14,000 $\mathrm{g}$ for 10 minutes. Fragmentation of chromatin was evaluated on extracted DNA using either: 1) a c-Kit enriched 500,000 cell sample (see above) processed in parallel (one of six tubes sonicated simultaneously) and agarose gel electrophoresis 
or, 2) direct sample size inspection of a $20 \mathrm{ul}$ aliquot using a Bioanalyzer (Agilent DNA1000 kit cat\#50671504) (Additional file 2: Figure S2). Output quantity was determined for each sample using the Qubit instrument (Invitrogen, HS-assay cat\#Q32851).

\section{Chromatin Immunoprecipitation}

ChIP was performed essentially as described previously with ca. 125,000 cells for histone mark and ca. 250,000 cells for CEBPA ChIP [30]. Quanta of used antibodies (CEBPA, Santa Cruz sc-61, lot\#J0407, 0.2 ug; H3K27me3, Cell signaling \#9733S, lot\#2, 1 ul; H3K4me3, Cell signaling \#9751S, lot\#2, $1 \mathrm{ul}$ ) and protein A beads (Sigma, cat\#P9424, $10 \mathrm{ul}$ $50 \% / 50 \%$ beads/RIPA-low salt $(140 \mathrm{mM}))$ were optimized for low input amounts, using siliconized tubes (Biozym, cat\#1267-2970). Preincubation was performed with $10 \mathrm{ul}$ of bead-slurry to minimize background. Washing conditions and buffers as in [30], but with 5 minute, $500 \mu \mathrm{l}$ washes; $2 \times$ RIPA-low salt $(140 \mathrm{mM} \mathrm{NaCl})$ and $2 \times$ RIPA-high salt (500 mM NaCl) for CEBPA ChIP and $1 \times$ RIPA-low salt and $3 \times$ RIPA-high salt for the histone mark antibodies, replacing previous RIPA buffer washes. Retrieval of immuneprecipitated DNA was optimized using overnight proteinase $\mathrm{K}$ treatment and 6-hour $65^{\circ} \mathrm{C}$ de-crosslinking with phenol-chloroform (cat\#9732) extraction in phaselock tubes (5-prime, cat\#713-2536) to Lo-Bind tubes (Eppendorf, cat\#525-0130) as described [30]. Pico-scale ChIP DNA concentrations were determined using the fluorescent Nanodrop 3300 PicoGreen assay (ThermoScientific and Invitrogen, cat\#p7589) (Additional file 4: Figure S4). Quantitative PCR (qPCR) for ChIP validation was performed on a Roche Lightcycler 480 with primers amplifying known CEBPA target sequences or regions expected to be marked by the H3K4me3 or H3K27me3 histone modifications, comparing to predicted negative regions. Primers and ChIP enrichments are found in additional files (Additional file 12: Table S3 and Additional file 3: Figure S3). Full protocol and buffer recipes are included in additional files (Additional file 8: Additional Protocols and Buffer Recipes).

\section{Preparation of libraries from nano- and picogram input DNA}

Amplification of 2 ng ChIP DNA was essentially performed as described by the manufacturer (NEB, cat\#E6240S), with the use of precast $2 \%$ SYBR agarose gels (Invitrogen, cat\#G5218-02) and excision of band size 175-400 bp. Key modifications consisted of a 30 minute ligation step, $30 \mathrm{mi}-$ nute gel solubilization at $37^{\circ} \mathrm{C}$ of excised gel fragments, and a prolonged, double run-through elution step (each three minutes) with preheated $\left(55^{\circ} \mathrm{C}\right)$ elution buffer for all column purifications (Qiagen, cats\#28104,28704,28004) to ensure robust recovery. Amplification of picogram precipitated DNA was performed by adding carrier up to a total of $500 \mathrm{pg}, 1000 \mathrm{pg}$ or $2 \mathrm{ng}$ as indicated using purified, chromosomal E. coli DNA, sonicated to a size distribution of 200-500 bp (Diagenode current protocols). All steps were performed in Lo-Bind tubes (Eppendorf, cat\#525-0130). Libraries were generated for duplex or triplex sequencing using a NEB kit (cat\#E7335S), and size distributions assessed by Bioanalyzer (Agilent, High Sensitivity kit, cat\#5067-4626), (Additional file 7: Figure S5 and Additional file 10: Figure S7). Full protocol included in additional files (Additional file 8: Additional Protocols and Buffer Recipes).

\section{Sequencing and mapping}

All libraries were single-end sequenced on the Illumina HiSeq2000 platform at the Danish National High-throughput DNA Sequencing Centre. The resulting 50-mer reads were mapped to the NCBI7/mm9 (Mus musculus) genome assembly using Bowtie v. 0.12 .8 with standard settings for unique mapping [32]. An overview of sequencing and mapping statistics is presented in (Additional file 5: Table S1). See additional files for mapping of bacterial carrier sequences (Additional file 6: Additional Methods).

\section{Visualization, statistical analysis and validation of profiles}

Bigwig files for UCSC genome coverage visualization were generated using Samtools [33,34], Bedtools [35], and UCSC wigtobigwig [36]. All coverage heatmaps were built using SeqMiner [37]. Histone mark profiles were examined at transcription start site (TSSs) regions (1000 bp) based on RefSeq longest isoform gene positions [38], excluding noncoding and mitochondrial genes. Peakcalling was performed using MACS2, with parameter "-to-large" [39]. A hematopoietic progenitor IgG mock ChIP-seq data set of 31,088,767 mapped reads was used as reference [27]. CEBPA-1 peak regions for analysis were defined by MACS2 cut-off of fold 30, q-value 50. For comparisons, CEBPA-1 and -2/-3/-4/-5/-6 coverages were normalized to mapped read depth. De novo motif search was performed with MEME-ChIP online using CEBPA-1 200 bp peak regions centered on summits [40]. Centrimo was used for distribution analysis of enriched motifs [41]. See additional files for genomic position annotations of reads and peaks, ChIP track coverage/ gene expression correlations and CEBPA-1 peak region Phylo-P conservation analysis (Additional file 6: Additional Methods).

\section{Availability of supporting data}

Supporting data is included in Additional files 1, 2, 3, 4, 5, 6, 7, 8, 9, 10, 11, 12 and 13 and at NCBI Gene Expression Omnibus (GEO), entry: GSE55850. 


\section{Additional files}

Additional file 1: Figure S1. Schematic representation of fluorescence activated cell sorting strategy. This is a depiction of the FACS gating regimen used. Detailed description is provided within the file.

Additional file 2: Figure S2. Validation of sonicated input chromatin. A demonstration of gel-electroforetic and Bioanalyzer analyses of input chromatin DNA size distribution. Detailed description is provided within the file.

Additional file 3: Figure S3. Assessment of individual sample ChIP enrichment. This is a figure showing $\mathrm{QPCR}$ validation of ChIP enrichments prior to library generation. Detailed description is provided within the file.

Additional file 4: Figure S4. Measuring picograms of DNA with the fluorescence Nanodrop 3300. A figure depicting fluorescent nanodrop measurement linearity and reproducibility in the $5-400 \mathrm{pg}$ range. Detailed description is provided within the file.

Additional file 5: Table S1. Sequencing and mapping statistics. Detailed description is provided within the file.

Additional file 6: Additional methods. This document is a description of additional methods including mapping of bacterial carrier DNA, genomic annotation of peaks/mapped reads, promoter coverage correlation with gene expression and conservation analysis.

Additional file 7: Figure S5. Size distributions of amplified ChIP-DNA libraries. Libraries assessed by Bioanalyzer analysis. Detailed description is provided within the file.

Additional file 8: Additional protocols. This contains detailed protocols for small-scale ChIP, amplification methodology and buffer recipes.

Additional file 9: Figure S6. Validation of sonicated input chromatin size distribution from limited cell numbers. Detailed description is provided within the file.

Additional file 10: Figure S7. Illumina sequencing libraries done with or without bacterial carrier. Detailed description is provided within the file.

Additional file 11: Table S2. List of comparable low-cell number datasets with NGS-QC scores. Detailed description is provided within the file.

Additional file 12: Table S3. List of primer sets used. Sequences of all primers used for this study.

Additional file 13: Additional references. This is a list of references used in additional methods and protocols.

\section{Abbreviations}

ChIP-seq: Chromatin ImmunoPrecipitation with deep sequencing; CEBPA: CCAAT/ enhancer binding protein (C/EBP), alpha, aka C/EBPa; H3K4me3: H3K27me3, Histone modifications H3 Lysine4-trimethyl and H3 Lysine27-trimethyl; MACS: Model-based analysis of ChIP-Seq data; TF: Transcription factor

\section{Competing interests}

The authors declare that they have no competing interests.

\section{Authors' contributions}

JSJ and BTP conceived the study. Experimental work was done by JSJ, MSH, MBS and A-KF. Bioinformatics and statistical analysis was conducted by JSJ, FOB, JW and KV-S. Figures were made by JSJ and FOB. The manuscript was written by JSJ and edited by FOB and BTP. All authors read and approved the final manuscript.

\section{Acknowledgements}

We thank Anna Fossum for expert help with fluorescence activated cell sorting. This study was supported by grants from The Novo Nordisk Foundation, the Danish Cancer Society and through a center grant from the Novo Nordisk Foundation (The Novo Nordisk Foundation Section for Stem Cell Biology in Human Disease).

\section{Author details}

${ }^{1}$ The Finsen Laboratory, Rigshospitalet, Faculty of Health Sciences, University of Copenhagen, Ole Maaløes Vej 5, 2200 Copenhagen, Denmark. ²Biotech Research and Innovation Centre (BRIC), University of Copenhagen, Ole Maaløes Vej 5, 2200 Copenhagen, Denmark. ${ }^{3}$ Danish Stem Cell Center (DanStem), Faculty of Health Sciences, University of Copenhagen, 3B Blegdamsvej, 2200 Copenhagen, Denmark. ${ }^{4}$ The Bioinformatics Centre, Department of Biology, Faculty of Natural Sciences, University of Copenhagen, Ole Maaløes Vej 5, 2200 Copenhagen, Denmark. ${ }^{5}$ Present address: Danish Pediatric Asthma Center, Copenhagen University Hospital Gentofte, Ledreborg Alle 34, 2820 Gentofte, Denmark.

\section{Received: 25 November 2014 Accepted: 22 December 2014}

Published online: 05 February 2015

\section{References}

1. Furey TS. ChIP-seq and beyond: new and improved methodologies to detect and characterize protein-DNA interactions. Nat Rev Genet. 2012;13:840-52.

2. Park PJ. ChIP-seq: advantages and challenges of a maturing technology. Nat Rev Genet. 2009;10:669-80.

3. Dahl JA, Collas P. MicroChIP-a rapid micro chromatin immunoprecipitation assay for small cell samples and biopsies. Nucleic Acids Res. 2008;36:e15.

4. Zhang JA, Mortazavi A, Williams BA, Wold BJ, Rothenberg EV. Dynamic transformations of genome-wide epigenetic marking and transcriptional control establish T cell identity. Cell. 2012;149:467-82.

5. Adli M, Zhu J, Bernstein BE. Genome-wide chromatin maps derived from limited numbers of hematopoietic progenitors. Nat Methods. 2010;7:615-8.

6. Shankaranarayanan P, Mendoza-Parra MA, Walia M, Wang L, Li N, Trindade LM, et al. Single-tube linear DNA amplification (LinDA) for robust ChIP-seq Nat Methods. 2011:8:565-7.

7. Lara-Astiaso D, Weiner A, Lorenzo-Vivas E, Zaretsky I, Jaitin DA, David E, et al. Immunogenetics. Chromatin state dynamics during blood formation. Science. 2014;345:943-9.

8. Pronk CJ, Rossi DJ, Mansson R, Attema JL, Norddahl GL, Chan CK, et al. Elucidation of the phenotypic, functional, and molecular topography of a myeloerythroid progenitor cell hierarchy. Cell Stem Cell. 2007:1:428-42.

9. Cleaves R, Wang QF, Friedman AD. C/EBPalphap30, a myeloid leukemia oncoprotein, limits G-CSF receptor expression but not terminal granulopoiesis via site-selective inhibition of C/EBP DNA binding. Oncogene. 2004;23:716-25.

10. Kirstetter $P$, Schuster MB, Bereshchenko O, Moore S, Dvinge H, Kurz E, et al. Modeling of C/EBPalpha mutant acute myeloid leukemia reveals a common expression signature of committed myeloid leukemia-initiating cells. Cancer Cell. 2008:13:299-310.

11. Bereshchenko O, Mancini E, Moore S, Bilbao D, Mansson R, Luc S, et al. Hematopoietic stem cell expansion precedes the generation of committed myeloid leukemia-initiating cells in C/EBPalpha mutant AML. Cancer Cell. 2009:16:390-400.

12. Hasemann MS, Damgaard I, Schuster MB, Theilgaard-Monch K, Sorensen AB, Mrsic A, et al. Mutation of C/EBPalpha predisposes to the development of myeloid leukemia in a retroviral insertional mutagenesis screen. Blood. 2008;111:4309-21.

13. Li B, Carey M, Workman JL. The role of chromatin during transcription. Cell. 2007;128:707-19.

14. Bernstein BE, Humphrey EL, Erlich RL, Schneider R, Bouman P, Liu JS, et al. Methylation of histone $\mathrm{H} 3$ Lys 4 in coding regions of active genes. Proc Natl Acad Sci U S A. 2002:99:8695-700.

15. Schneider R, Bannister AJ, Myers FA, Thorne AW, Crane-Robinson C, Kouzarides T. Histone $\mathrm{H} 3$ lysine 4 methylation patterns in higher eukaryotic genes. Nat Cell Biol. 2004;6:73-7.

16. Min J, Zhang $Y, X u$ RM. Structural basis for specific binding of Polycomb chromodomain to histone $\mathrm{H} 3$ methylated at Lys 27. Genes Dev. 2003;17:1823-8.

17. Fischle W, Wang Y, Jacobs SA, Kim Y, Allis CD, Khorasanizadeh S. Molecular basis for the discrimination of repressive methyl-lysine marks in histone $\mathrm{H} 3$ by Polycomb and HP1 chromodomains. Genes Dev. 2003;17:1870-81.

18. De Gobbi M, Garrick D, Lynch M, Vernimmen D, Hughes JR, Goardon N, et al. Generation of bivalent chromatin domains during cell fate decisions. Epigenetics Chromatin. 2011;4:9.

19. Pietersen $A M$, van Lohuizen M. Stem cell regulation by polycomb repressors: postponing commitment. Curr Opin Cell Biol. 2008;20:201-7. 
20. Consortium EP, Bernstein BE, Birney E, Dunham I, Green ED, Gunter C, et al. An integrated encyclopedia of DNA elements in the human genome. Nature. 2012;489:57-74.

21. Jakobsen JS, Waage J, Rapin N, Bisgaard HC, Larsen FS, Porse BT. Temporal mapping of CEBPA and CEBPB binding during liver regeneration reveals dynamic occupancy and specific regulatory codes for homeostatic and cell cycle gene batteries. Genome Res. 2013;23:592-603.

22. Heliot C, Cereghini S. Analysis of in vivo transcription factor recruitment by chromatin immunoprecipitation of mouse embryonic kidney. Methods Mol Biol. 2012;886:275-91.

23. Li XY, Biggin MD. Genome-wide in vivo cross-linking of sequence-specific transcription factors. Methods Mol Biol. 2012;809:3-26.

24. Zwart W, Koornstra R, Wesseling J, Rutgers E, Linn S, Carroll JS. A carrier-assisted ChIP-seq method for estrogen receptor-chromatin interactions from breast cancer core needle biopsy samples. BMC Genomics. 2013;14:232.

25. Mendoza-Parra MA, Van Gool W, Mohamed Saleem MA, Ceschin DG, Gronemeyer $\mathrm{H}$. A quality control system for profiles obtained by ChIP sequencing. Nucleic Acids Res. 2013;41:e196.

26. Ohlsson E, Hasemann MS, Willer A, Lauridsen FK, Rapin N, Jendholm J, et al. Initiation of MLL-rearranged AML is dependent on C/EBPalpha. J Exp Med. 2014;211:5-13

27. Hasemann MS, Lauridsen FK, Waage J, Jakobsen JS, Frank AK, Schuster MB et al. C/EBPalpha Is Required for Long-Term Self-Renewal and Lineage Priming of Hematopoietic Stem Cells and for the Maintenance of Epigenetic Configurations in Multipotent Progenitors. PLoS Genet. 2014;10:e1004079.

28. Fullwood MJ, Liu MH, Pan YF, Liu J, Xu H, Mohamed YB, et al. An oestrogen-receptor-alpha-bound human chromatin interactome. Nature. 2009:462:58-64.

29. O'Neill LP, VerMilyea MD, Turner BM. Epigenetic characterization of the early embryo with a chromatin immunoprecipitation protocol applicable to small cell populations. Nat Genet. 2006;38:835-41.

30. Sandmann T, Jakobsen JS, Furlong EE. ChIP-on-chip protocol for genome-wide analysis of transcription factor binding in Drosophila melanogaster embryos. Nat Protoc. 2006;1:2839-55.

31. Schuster MB, Frank AK, Bagger FO, Rapin N, Vikesaa J, Porse BT. Lack of the p42 form of C/EBPalpha leads to spontaneous immortalization and lineage infidelity of committed myeloid progenitors. Exp Hematol. 2013;41:882-93. e816.

32. Langmead B, Trapnell C, Pop M, Salzberg SL. Ultrafast and memory-efficient alignment of short DNA sequences to the human genome. Genome Biol. 2009; 10:R25.

33. Kent WJ, Sugnet CW, Furey TS, Roskin KM, Pringle TH, Zahler AM, et al. The human genome browser at UCSC. Genome Res. 2002;12:996-1006.

34. Li H, Handsaker B, Wysoker A, Fennell T, Ruan J, Homer N, et al. The Sequence Alignment/Map format and SAMtools. Bioinformatics. 2009;25:2078-9.

35. Quinlan AR, Hall IM. BEDTools: a flexible suite of utilities for comparing genomic features. Bioinformatics. 2010;26:841-2.

36. Kent WJ, Zweig AS, Barber G, Hinrichs AS, Karolchik D. BigWig and BigBed: enabling browsing of large distributed datasets. Bioinformatics. 2010;26:2204-7.

37. Ye T, Krebs AR, Choukrallah MA, Keime C, Plewniak F, Davidson I, et al. seqMINER: an integrated ChIP-seq data interpretation platform. Nucleic Acids Res. 2011;39:e35.

38. Pruitt KD, Tatusova T, Brown GR, Maglott DR. NCBI Reference Sequences (RefSeq): current status, new features and genome annotation policy. Nucleic Acids Res. 2012;40:D130-5.

39. Zhang Y, Liu T, Meyer CA, Eeckhoute J, Johnson DS, Bernstein BE, et al. Model-based analysis of ChIP-Seq (MACS). Genome Biol. 2008:9:R137.

40. Machanick P, Bailey TL. MEME-ChIP: motif analysis of large DNA datasets. Bioinformatics. 2011;27:1696-7.

41. Bailey TL, Machanick P. Inferring direct DNA binding from ChIP-seq. Nucleic Acids Res. 2012;40:e128.

\section{Submit your next manuscript to BioMed Central and take full advantage of:}

- Convenient online submission

- Thorough peer review

- No space constraints or color figure charges

- Immediate publication on acceptance

- Inclusion in PubMed, CAS, Scopus and Google Scholar

- Research which is freely available for redistribution

Submit your manuscript at www.biomedcentral.com/submit 